\title{
Trends of reactive hyperaemia responses to repetitive loading on skin tissue of rats - Implications for pressure ulcer prevention
}

\begin{abstract}
Tissue recovery is important in preventing tissue deterioration, which is induced by pressure and may lead to pressure ulcers (PU). Reactive hyperaemia (RH) is an indicator used to identify people at risk of PU. In this study, the effect of different recovery times on RH trend is investigated during repetitive loading. Twenty-one male Sprague-Dawley rats (seven per group), with body weight of 385-485 g, were categorised into three groups and subjected to different recovery times with three repetitive loading cycles. The first, second, and third groups were subjected to short ( $3 \mathrm{~min}$ ), moderate (10 $\mathrm{min}$ ), and prolonged (40 $\mathrm{min}$ ) recovery, respectively, while fixed loading time and pressure $(10 \mathrm{~min}$ and $50 \mathrm{mmHg}$, respectively). Peak hyperaemia was measured in the three cycles to determine trends associated with different recovery times. Three RH trends (increasing, decreasing, and inconsistent) were observed. As the recovery time is increased ( $3 \mathrm{~min}$ vs. $10 \mathrm{~min}$ vs. $40 \mathrm{~min}$ ), the number of samples with increasing RH trend decreases (57\% vs. $29 \%$ vs. $14 \%$ ) and the number of samples with inconsistent RH trend increases (29\% vs. $57 \%$ vs. $72 \%$ ). All groups consists of one sample with decreasing RH trend (14\%). Results confirm that different recovery times affect the RH trend during repetitive loading. The $\mathrm{RH}$ trend may be used to determine the sufficient recovery time of an individual to avoid PU development.
\end{abstract}

Keyword: Reactive hyperaemia trend; Repetitive loading; Pressure ulcer; Tissue recovery; Recovery time 